\title{
Nonoperative Treatment of Appendicitis during Pregnancy in a Remote Area
}

\author{
Anne-Kathrine Carstens, MD ${ }^{1}$ Lise Fensby, MD ${ }^{1} \quad$ Luit Penninga, MD, $\mathrm{PhD}^{1}$ \\ ${ }^{1}$ Department of Surgery, Ilulissat Hospital, Ilulissat, Greenland \\ Address for correspondence Luit Penninga, MD, PhD, llulissat \\ Hospital, Avannaa Health Region, Postbox 514, llulissat 3952, \\ Am J Perinatol Rep 2018;8:e37-e38. \\ Greenland (e-mail: LP@ctu.dk).
}

\begin{abstract}
Appendicitis is the most common nonobstetric surgical disease during pregnancy. Appendicitis during pregnancy is associated with an increased risk of morbidity and perforation compared with the general population. Furthermore, it may cause preterm birth and fetal loss, and quick surgical intervention is the established treatment option in pregnant women with appendicitis. In Greenland, geographical distances are very large, and weather conditions can be extreme, and surgical care is not always

Keywords

- appendicitis

- pregnancy

- antibiotics

- bridge-to-surgery

- nonoperative treatment

- appendectomy immediately available. Hence, antibiotic treatment is often initiated as a bridge-tosurgery. We report on a pregnant Greenlandic Inuit woman with appendicitis who was treated with intravenous antibiotics. Antibiotic treatment was successful before surgical care became available and the patient was not operated. No complications occurred, and further pregnancy was uneventful. Our case suggests that antibiotic treatment of appendicitis during pregnancy as a bridge-to-surgery may be a sensible treatment option in remote areas, where no surgical care is immediately available. In some cases, antibiotic treatment may turn out to be definitive treatment.
\end{abstract}

Appendicitis is the most common nonobstetric surgical diseases during pregnancy. ${ }^{1-4}$ Appendicitis during pregnancy is difficult to diagnose. ${ }^{3-5}$ This is due to rather unspecific symptoms associated with normal pregnancy like abdominal pain, nausea, and vomiting. In addition, leukocytosis is a normal phenomenon in pregnant women. ${ }^{3,4}$ The diagnosis of appendicitis based on clinical examination can be supported by different imaging technics. ${ }^{6}$ Ultrasonography is the first choice in pregnant women. If ultrasound is inconclusive, magnetic resonance imaging is the next step if available. Also low-dose computed tomography has been applied. ${ }^{6}$

As a consequence of the diagnostic difficulties, perforation rates are higher in pregnant women compared with the nonpregnant population. ${ }^{3}$ Perforation increases the risk of morbidity and the risk of preterm delivery and fetal loss. ${ }^{7}$ Hence, early surgical intervention is the recommended treatment strategy for appendicitis during pregnancy. ${ }^{2,7}$

Greenland is the largest island in the world, and geographical distances are large. Broadest east-west distance is 652 miles $(1050 \mathrm{~km})$, and north-south distance is 1647 miles $(2650 \mathrm{~km})$. In addition, weather conditions can be extreme,

received

March 26, 2017

accepted

December 5, 2017 and the population density is very low. Hence, access to health care facilities where appropriate surgical care can be provided is not always instantly available. This may necessitate the need for alternative treatment options.

In nonpregnant adults, nonoperative treatment with antibiotics is an alternative treatment option for appendicitis. ${ }^{8}$ We have previously reported on antibiotic treatment of appendicitis during pregnancy as a bridge-to-surgery in remote areas. ${ }^{7}$ In this case, we report on a pregnant Greenlandic Inuit woman with appendicitis who is successfully treated with antibiotics without surgical intervention.

\section{Case}

A 34-year-old woman presented during the second trimester of pregnancy (a gravida 4 para 2) with abdominal pain in the right iliac fossa, fever, nausea and vomiting, and was admitted for suspicion of appendicitis.

The patient had pain in the right iliac fossa with referred tenderness. Transabdominal ultrasonography was performed and showed an appendix with thickened walls. Laboratory
Copyright $\odot 2018$ by Thieme Medical Publishers, Inc., 333 Seventh Avenue, New York, NY 10001, USA. Tel: +1(212) 584-4662.
License terms

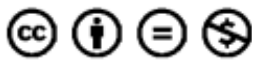

10.1055/s-0037-1620279. ISSN 2157-6998. 
testing was performed and revealed leukocytosis $\left(17 \times 10^{9} / \mathrm{L}\right.$ on admission decreasing to $7 \times 10^{9} / \mathrm{L}$ on day 3 ) and increased C-reactive protein $(9 \mathrm{mg} / \mathrm{L}$ on admission, $39 \mathrm{mg} / \mathrm{L}$ on day 2 , and $27 \mathrm{mg} / \mathrm{L}$ on day 3 when the patient was discharged).

The differential diagnosis of acute appendicitis during pregnancy includes both obstetric and nonobstetric diseases. In this case, transvaginal ultrasonography was used to exclude obstetric problems. Hydronephrosis and other urological conditions were excluded with abdominal ultrasonography and urinary examination.

The patient was treated conservatively with antibiotics and fluids as no surgical care was instantly available, and due to extreme weather conditions transfer of the patient was not possible. Two different antibiotics were used: metronidazole $500 \mathrm{mg}$ three times daily and ceftriaxone $1 \mathrm{~g}$ once daily. Treatment was administered intravenously for 3 days followed by 4 days with oral treatment. The total duration of antibiotic treatment was 7 days.

After 3 days of observation and intravenous antibiotic treatment, the patient had improved clinically; the abdominal pain had disappeared, and she was discharged. Further pregnancy was uneventful, and a healthy child was born by vaginal delivery after 40 weeks of gestation.

\section{Discussion}

During decades, the treatment of acute uncomplicated appendicitis has been appendectomy. ${ }^{8}$ This applies to both the pregnant and nonpregnant population. ${ }^{2,8}$ In pregnant women, early surgical intervention is especially important because of the possible severe consequences of perforation. ${ }^{2}$ Recent trials have shown that nonoperative management with intravenous antibiotics is a valuable alternative for the treatment of acute uncomplicated appendicitis in nonpregnant patients, when compared with surgical appendectomy. ${ }^{8}$ In nonpregnant adults, antibiotic treatment can avoid the need for surgery in the majority of patients with uncomplicated appendicitis, and less complications were observed with nonoperative treatment compared with appendectomy. ${ }^{8}$

Antibiotic treatment has been applied as a valuable treatment option for adult nonpregnant patients with acute appendicitis in both remote and nonremote areas. ${ }^{8}$ The question arises, whether pregnant women who develop acute appendicitis, and who live in a remote area where no surgical care is instantly available, should start intravenous antibiotic treatment. We present a case of appendicitis in a pregnant woman managed successfully with antibiotics. Yefet et al have previously presented a similar case with a pregnant woman who denied surgery, and was treated with gentamicin and metronidazole intravenously for 3 days followed by oral amoxicillin/clavulanic acid for 14 days. $^{9}$
Another group has reported on two pregnant women with a perforated appendix who were treated nonoperatively with antibiotics. ${ }^{10}$ One of these two pregnant women recovered uneventfully, and the other one had a recurrence of the appendicitis. This recurrence was again treated successfully with antibiotics at 32 weeks of gestation, though she delivered preterm at 34 weeks of gestation. ${ }^{10}$

Similar to nonpregnant adults, symptoms and signs of acute appendicitis in some pregnant patients treated with intravenous antibiotics may completely resolve, and the need for surgical treatment might disappear.

In conclusion, this case suggests that it may be possible to manage acute uncomplicated appendicitis during pregnancy nonoperatively. This is relevant in remote areas where no surgical care is instantly available. This could be of relevance as well if the patient denies surgery.

Whether antibiotic treatment can avoid the need for surgical intervention in pregnant patients similar to nonpregnant adults and, even more important, reduce complications is unclear. Furthermore, outside of remote area, it is unclear whether the benefits of nonoperative treatment outweigh the risk of surgical delay.

\section{References}

1 Coleman MT, Trianfo VA, Rund DA. Nonobstetric emergencies in pregnancy: trauma and surgical conditions. Am J Obstet Gynecol 1997;177(03):497-502

2 Tamir IL, Bongard FS, Klein SR. Acute appendicitis in the pregnant patient. Am J Surg 1990;160(06):571-575, discussion 575576

3 Andersen B, Nielsen TF. Appendicitis in pregnancy: diagnosis, management and complications. Acta Obstet Gynecol Scand 1999;78(09):758-762

4 Al-Mulhim AA. Acute appendicitis in pregnancy. A review of 52 cases. Int Surg 1996;81(03):295-297

5 Richards C, Daya S. Diagnosis of acute appendicitis in pregnancy. Can J Surg 1989;32(05):358-360

6 Smith MP, Katz DS, Lalani T, et al. ACR appropriateness Criteria ${ }^{\circledR}$ right lower quadrant pain-suspected appendicitis. Ultrasound Q 2015;31(02):85-91

7 Dalsgaard Jensen T, Penninga L. Appendicitis during pregnancy in a Greenlandic Inuit woman; antibiotic treatment as a bridge-to-surgery in a remote area. BMJ Case Rep 2016;2016:bcr2016214722. Doi: 10.1136/bcr-2016-214722

8 Varadhan KK, Neal KR, Lobo DN. Safety and efficacy of antibiotics compared with appendicectomy for treatment of uncomplicated acute appendicitis: meta-analysis of randomised controlled trials. BMJ 2012;344:e2156

9 Yefet E, Romano S, Chazan B, Nachum Z. Successful treatment of acute uncomplicated appendicitis in pregnancy with intravenous antibiotics. Eur J Obstet Gynecol Reprod Biol 2013;169(01): $121-122$

10 Young BC, Hamar BD, Levine D, Roqué H. Medical management of ruptured appendicitis in pregnancy. Obstet Gynecol 2009;114 (2 Pt 2):453-456 